Louisiana State University

LSU Digital Commons

$12-7-2010$

\title{
Methodology for determining doses to in-field, out-of-field and partially in-field organs for late effects studies in photon radiotherapy
}

\author{
Rebecca M. Howell \\ University of Texas Graduate School of Biomedical Sciences at Houston \\ Sarah B. Scarboro \\ University of Texas Graduate School of Biomedical Sciences at Houston \\ Phillip J. Taddei \\ University of Texas MD Anderson Cancer Center \\ Sunil Krishnan \\ University of Texas MD Anderson Cancer Center \\ Stephen F. Kry \\ University of Texas Graduate School of Biomedical Sciences at Houston
}

See next page for additional authors

Follow this and additional works at: https://digitalcommons.Isu.edu/physics_astronomy_pubs

\section{Recommended Citation}

Howell, R., Scarboro, S., Taddei, P., Krishnan, S., Kry, S., \& Newhauser, W. (2010). Methodology for determining doses to in-field, out-of-field and partially in-field organs for late effects studies in photon radiotherapy. Physics in Medicine and Biology, 55 (23), 7009-7023. https://doi.org/10.1088/0031-9155/ $55 / 23 /$ S04 


\section{Authors}

Rebecca M. Howell, Sarah B. Scarboro, Phillip J. Taddei, Sunil Krishnan, Stephen F. Kry, and Wayne D. Newhauser 


\title{
Methodology for determining doses to in-field, out-of-field and partially in-field organs for late effects studies in photon radiotherapy
}

\author{
Rebecca M Howell ${ }^{1,2,4}$, Sarah B Scarboro ${ }^{1,2}$, Phillip J Taddei ${ }^{2}$, Sunil Krishnan ${ }^{3}$, Stephen \\ F Kry $^{1,2}$, and Wayne D Newhauser ${ }^{1,2}$ \\ ${ }^{1}$ Graduate School of Biomedical Sciences, The University of Texas Health Science Center, 6767 \\ Bertner Avenue, Houston, TX 77030, USA \\ 2Department of Radiation Physics, Unit 94, The University of Texas MD Anderson Cancer Center, \\ 1515 Holcombe Boulevard, Houston, TX 77030, USA \\ ${ }^{3}$ Department of Radiation Oncology, Unit 97, The University of Texas MD Anderson Cancer \\ Center, 1515 Holcombe Boulevard, Houston, TX 77030, USA
}

\begin{abstract}
An important but little examined aspect of radiation dosimetry studies involving organs outside the treatment field is how to assess dose to organs that are partially within a treatment field; this question is particularly important for studies intended to measure total absorbed dose in order to predict the risk of radiogenic late effects, such as second cancers. The purpose of this investigation was therefore to establish a method to categorize organs as in-field, out-of-field or partially infield that would be applicable to both conventional and modern radiotherapy techniques. In this study, we defined guidelines to categorize the organs based on isodose inclusion criteria, developed methods to assess doses to partially in-field organs, and then tested the methods by applying them to a case of intensity-modulated radiotherapy for hepatocellular carcinoma based on actual patient data. For partially in-field organs, we recommend performing a sensitivity test to determine whether potential inaccuracies in low-dose regions of the DVH (from the treatment planning system) have a substantial effect on the mean organ dose, i.e. $>5 \%$. In such cases, we suggest supplementing calculated DVH data with measured dosimetric data using a volumeweighting technique to determine the mean dose.
\end{abstract}

\section{Introduction}

Studies evaluating late effects, such as second cancer risk, require accurate knowledge of both primary and stray radiation in order to accurately estimate the risk to organs throughout the body (Boice et al 1985, Stovall et al 1989, 2006, Bhatia et al 2003, Ron 2003, Rubino et al 2003a, 2003b, Lin and Teitell 2005, Newhauser et al 2009). For organs within a treatment field, radiation doses are relatively high and attributed mainly to primary radiation. As the distance from the treatment field increases and the organs become peripheral to the treatment field, the doses become progressively lower and are attributed mainly to stray radiation (Stovall et al 1995).

(C) 2010 Institute of Physics and Engineering in Medicine

${ }^{4}$ Author to whom any correspondence should be addressed. rhowell@ mdanderson.org. 
In-field radiation doses can be accurately and rapidly calculated using commercially available treatment planning systems (TPSs) (Aspradakis et al 2003, Van Esch et al 2006). These TPSs do not, however, accurately model doses outside the treatment field, nor are they commissioned for such calculations (Das et al 2008). A recent study evaluated the accuracy with which a commercial TPS calculated absorbed dose in regions where the isodose lines reported by the TPS were less than 5\% of the prescribed dose (Howell et al 2010). That study demonstrated that in this very low stray dose region, the predicted doses were at worst $60 \%$ lower than corresponding measured data and that the accuracy of the TPS calculated doses decreased with increasing distance from the treatment field. An important consideration in determining peripheral organ doses from stray radiation is the definition of the field border. In conventional photon radiation therapy, out-of-field organs are easily defined by their proximity to the field border which is defined by the collimating jaws. However, for modern photon radiation therapy where fields are defined by modulated beam intensities it is difficult to definitively define a field border. Dose calculations by a TPS may also be precluded by a lack of imaging information related to the organs in question: patients' computed tomography (CT) scans typically include only the anatomy required for treatment planning and nearby organs or structures at risk, in keeping with the best practices of avoiding unnecessary exposure (Goske et al 2009). These datasets typically extend only a few centimeters above and below the target volume. Most treatment planning CT scans therefore contain incomplete information on peripheral organs that nevertheless receive lowdose stray radiation during treatment.

Measurements obtained using thermoluminescent dosimeters (TLDs) in an anthropomorphic phantom are widely used for determining peripheral organ doses from photon radiation therapy. Measurements in phantom are accurate over a broad range of doses and closely reproduce the irradiation of a patient. In addition, measurement techniques are not limited by the extent of CT datasets. Radiation dose measurements in anthropomorphic phantoms are considered the gold standard in peripheral dose assessment and have frequently been used to determine peripheral organ doses in studies of radiation-induced late effects from photon radiotherapy (Stovall et al 1989, 2006, Carr et al 2002, Meeks et al 2002, Sigurdson et al 2005, Klein et al 2006, Reft et al 2006, Xu et al 2008). However, measurements in phantom are very time consuming and require special measurement equipment. Computational techniques such as Monte Carlo modeling are an alternative approach to measurements for determining peripheral organ doses. Such simulations can accurately calculate peripheral organ dose, provided the model is benchmarked for out-of-field doses (Kry et al 2006, Bednarz and Xu 2009). However, this approach requires computing resources and the CT datasets should include the patient's complete anatomy or replace missing anatomy with phantom substitutes.

Although it is well understood how to calculate dose to organs inside the treatment field and how to measure or calculate dose to organs outside the treatment field, relatively little attention has been paid in the literature to dosimetry for organs that are partially within the treatment field. In particular, the accuracy of the TPS calculations for such organs, especially when reported in terms of mean organ dose, has not been described. Commercial TPSs may be inadequate for this task due to inaccuracies in the out-of-field component of the organ dose, but simulations and phantom studies are prohibitively time consuming and costly for routine use. Thus, there is a need for a method to determine when the TPSpredicted dose is adequate and when it is not. Such a method should be generally applicable to either conventional radiation therapy or modern radiation therapy with modulated beam intensities. This methodology will be particularly helpful when analyzing risk for second cancers and other late effects, where the total dose from both high-dose primary radiation and low-dose stray radiation must be known. Thus, the objectives of this investigation therefore were to establish a method to categorize organs as in-field, out-of-field or partially 
in-field that is applicable to both conventional and contemporary radiotherapy techniques; to evaluate the accuracy of the mean organ dose reported by a widely used TPS for organs partially within the treatment field; and to use this evaluation to establish a consistent methodology for determining doses to such organs in radiotherapy. The categorization of organs as in-field, out-of-field or partially in-field was based on isodose inclusion criteria rather than on proximity to the field border. We used dose volume histograms (DVHs) from the TPS to quantify mean organ doses for in-field organs and measurements in an anthropomorphic phantom to estimate mean doses for out-of-field organs. We then defined guidelines to evaluate the potential effect of inaccuracies in the low-dose components of DVHs for partially in-field organs to determine the effect on the mean organ doses reported by the TPS. This new methodology was tested on an example case for a patient receiving intensity-modulated radiotherapy (IMRT) for liver cancer. We chose this one case because IMRT is a complex photon therapy delivery technique and would allow us to rigorously test the methodology described in this study. We chose to focus this study on 6 MV because it is the most commonly used beam energy for IMRT. We choose this particular case because we had a full body CT scan allowing us to examine all three categories of organs including in field, out-of-field and partially in-field. Additionally, the treatment plan for this case included a wide range of doses including low doses. These low doses are of particular interest for late effects studies.

\section{Materials and methods}

\subsection{Treatment planning}

This study was carried out under an institutional review board-approved protocol for retrospective treatment planning studies. Dosimetric assessments and evaluations were based on a case of liver cancer treated at our institution. We selected this site because of our interest in comparing the risk of secondary malignant neoplasms from different treatment techniques for liver cancer (Taddei et al 2010). The planning CT scan was acquired using a $2.5 \mathrm{~mm}$ slice thickness and included anatomy from the head to mid-thigh for a man (age 59, height $174 \mathrm{~cm}$ and weight $93.9 \mathrm{~kg}$ ) who had been diagnosed with primary hepatocellular carcinoma.

Using a commercial TPS (Eclipse version 8.6, Varian Medical Systems, Palo Alto, CA), we developed an IMRT plan for this patient with $6 \mathrm{MV}$ photons. The anisotropic analytical algorithm with tissue heterogeneity corrections and a $2.5 \mathrm{~mm}$ calculation grid was used for dose calculations. The prescribed dose was $60 \mathrm{~Gy}$ in 30 fractions ( $2 \mathrm{~Gy} /$ fraction) with the criterion that $95 \%$ of the planning target volume was to receive $100 \%$ of the prescribed dose $\left(D_{95}=60 \mathrm{~Gy}\right)$. The treatment plan had nine dynamic multileaf collimator-shaped IMRT beams. The following structures were contoured for treatment planning: clinical target volume (CTV), liver, healthy liver (liver minus CTV), right (ipsilateral) kidney, left (contralateral) kidney, spinal cord, esophagus, lungs and stomach plus duodenum. Table 1 details the planning constraints and the final dosimetric treatment plan values. The final treatment plan was reviewed and approved by a board-certified radiation oncologist. In addition to the organs defined for treatment planning, other organs and tissues that are sensitive to radiogenic late effects were contoured, namely the bone marrow, bone surface, brain, eyes, thyroid, heart, breast, colon, bladder, rectum, prostate and testicles.

The treatment planning system (and the AAA dose calculation algorithm) used in this study was fully commissioned and validated for use in our clinic by American Board of Radiology certified medical physicists using the methodology described by Das et al (2008). The validation included comparisons of measured and calculated doses for both conventional and IMRT treatment plans within the treatment fields and penumbra (penumbra defined as the distance between the $90 \%$ and $10 \%$ isodoses). Additionally, the treatment planning system 
was credentialed by the Radiological Physics Center (RPC, Houston, TX) for the use of IMRT in clinical trials following RPC credentialing protocols (Ibbott et al 2006, Followill et al 2007). Out-of field dose assessment is typically not part of TPS commissioning and was not considered during the commissioning of the TPS used in this study. The Eclipse AAA models stray radiation using a finite-size virtual source with a Gaussian intensity distribution (Sievinen et al 2005).

\subsection{Measurement locations in the phantom}

In this study, we used a commercial male dosimetry phantom (ATOM, CIRS, Inc., Norfolk, VA) for dose measurements, figure 1. The phantom is transected horizontally in $2.5 \mathrm{~cm}$ slices and was custom fabricated to include a $1.5 \mathrm{~cm}^{2}$ grid of holes to hold TLD capsules as well as additional optimized TLD capsule locations for precise dosimetry in 20 internal organs. A CT scan of the phantom was acquired using a $2.5 \mathrm{~mm}$ slice thickness, which was then imported into the TPS. The phantom's organs were contoured using the image segmentation tools in the TPS and an organ map provided by the phantom manufacturer. The patient and phantom CT datasets were registered by manual vertebral body matching using registration tools within the TPS. After the CT datasets were registered, the patient's treatment plan was superimposed on the phantom CT dataset. The plan isocenter in the phantom was defined using the anterior and right lateral set-up fields from the patient's treatment plan.

The out-of-field measurement locations within the phantom were determined by selecting TLD locations that were within the organ map but outside the 5\% isodose line. The heart was also an out-of-field organ, but because the organ map does not include locations for the heart, TLD positions for this organ were defined independently by manual selection of locations within the $1.5 \mathrm{~cm}^{2}$ grid that correlated with structures in the heart based on visual inspection of the CT dataset.

The distance between the centroid of each out-of-field organ and the nearest 5\% isodose line was determined to evaluate the relationship between mean organ dose and distance from the treatment field edge. We used distance from the 5\% isodose line as a surrogate for distance from any particular field border because the treatment plan in this study included both obliquely incident and non-coplaner beams and thus it was difficult to define a single field edge. The coordinates of the center of each organ were determined using measurement tools in the TPS. Then we calculated the difference between the $z$-coordinate of the organ centroid and the $z$-coordinate of either the superior or inferior 5\% isodose line, as appropriate. This process is illustrated in figure 2, which shows the superior and inferior $5 \%$ isodose lines and the distances from them for an organ superior to the target volume (brain) and an organ below it (bladder). Similar measurements were made for each out-of-field organ.

\subsection{Measurements in the phantom}

Lithium fluoride TLD-100 powder capsules (Quantaflux Radiological Services, San Jose, CA) were selected for the in-phantom measurements. All TLD capsules were from the same batch to avoid batch-to-batch variations in sensitivity. The TLD capsules were loaded into the phantom at the positions specified above. The phantom was set up on a commercial medical linear accelerator (Varian Clinac 2100, Varian Medical Systems, Palo Alto, CA) treatment couch to the isocenter. The planned treatment fields were then delivered to the phantom as specified in the treatment plan. The linac used in this study is calibrated monthly for in-field dose using the American Association of Physicists in Medicine Task Group 51 protocol (Almond et al 1999). Additionally, for this study, the output was verified on the day of experimental measurements. 
Following irradiation, the TLDs were individually read using an established laboratory protocol consistent with Accredited Dosimetry Calibration Laboratory procedures, which accounts for energy response, linearity and dosimeter fading and has an uncertainty of $<3.0 \%$ (Kirby et al 1986, 1992). The TLD readings were converted to absorbed dose in tissue using a calibration coefficient from a set of reference TLDs (from the same batch) irradiated with Co-60. The Co-60 unit that was used to irradiate the TLD standards was certified and maintained by an Accredited Dosimetry Calibration Laboratory and was calibrated traceable to standards of the National Institute of Standards and Technology. The mean organ dose (and standard deviation) was calculated from all TLDs within each organ. All measured doses were normalized per absorbed dose delivered to the isocenter and are reported in Gray per prescribed Gray $\left(\mathrm{Gy} / \mathrm{Gy}_{\mathrm{Rx}}\right)$.

\subsection{Organ classification and methods for dose assessment for each organ category}

The definition of in-field organs is not as straightforward for advanced radiotherapies as it is for conventional techniques. In conventional radiotherapy, in-field organs are those that lie wholly within the regions defined by the collimating devices. For IMRT, conversely, the treatment fields are defined by modulated beam intensities rather than by static collimation. Consequently, we sought to define organs as in-field, out-of-field or partially in-field based on criteria other than distance from the field edge but that are generally applicable to both conventional radiotherapy and IMRT. Aspradakis et al (2003) found that doses reported by several modern TPSs are accurate within the treatment field and up to approximately $3 \mathrm{~cm}$ beyond the field edge. Additionally, in a recent study (Howell et al 2010) we found that the TPS used in this study underreported dose at distances greater than $3.5 \mathrm{~cm}$ beyond the field edge, corresponding to regions where the isodose lines were less than $5 \%$ of the prescribed dose. Based in part on the evidence from the above studies, we proposed to categorize organs as in-field, out-of-field or partially in-field using an isodose inclusion criterion, namely the $5 \%$ isodose level, as a conservative and generally applicable defining metric. We defined organs as being out-of-field if no part of the organ received more than 5\% of the prescribed dose. We defined organs as in-field if the entire organ (100\% of the volume) received more than $5 \%$ of the prescribed dose. We defined partially in-field organs as those that did not meet the in-field criterion but could not be classified as out-of-field. An example of two partially in-field organs, the lungs and left kidney, are shown in figure 3 . This figure is the frontal view of the patient CT dataset at the isocenter plane with the dose displayed as a color wash from the maximum dose of $112.9 \%$ to $0.1 \%$ of the prescribed dose. The $5 \%$ isodose line is outlined using a green contour and the out-of-field and in-field portions of the lungs and left kidney are shown in orange and white contours, respectively.

The mean doses for in-field organs were calculated using DVH data from the TPS, as is a standard practice in radiation treatment planning. The mean doses for out-of-field organs were determined using measured TLD measurements (described above in section 2.3). The mean doses to partially in-field organs were first calculated using DVH data from the TPS and then examined in terms of the sensitivity of the mean dose to inaccuracies in the lowdose component of the TPS data and adjusted accordingly (described below in section 2.5).

\subsection{Partially in-field organs: sensitivity analysis and volume-weighting technique}

Inaccuracy in low-dose regions may affect the low-dose component of DVH data for partially in-field organs. This inaccuracy may in turn affect the mean organ dose reported by the TPS, providing the rationale for investigating the potential effect of the low-dose component of the DVH data on the mean organ doses. Our previous finding of maximum difference of 60\% (Howell et al 2010) between doses reported by the TPS (used in this study) and measured doses in regions outside the 5\% isodose line prompted us to conduct a sensitivity analysis of how inaccuracy in the low-dose region of the DVH may affect the 
mean organ doses. Thus, we performed a sensitivity test to assess how much over- or underestimation of dose in the out-of-field region affects the reported mean dose of a partially in-field organ. Specifically, we calculated the mean organ doses after adjusting the absorbed dose by $+60 \%$ and $-60 \%$ for portions of the DVH with dose less than $5 \%$ of the prescribed dose. We selected $60 \%$ deviation to add a degree of conservatism to the sensitivity analysis. This method is illustrated in figure 4 for the two partially in-field organs shown in figure 3.

The adjusted mean doses were then compared to the mean doses reported by the TPS. If the adjusted means were within $\pm 5 \%$ of those reported by the TPS, then TPS data alone were used to determine the mean organ dose, otherwise we used a volume-weighting technique to calculate the mean organ dose described below. The 5\% criterion was chosen based on evidence that accuracy to within approximately $\pm 5 \%$ in dose delivery (95-105\% of the prescribed dose) is necessary for effective radiotherapy (ICRU 1987).

Volume-weighted mean organ doses were determined by taking a volume-weighted average of the in-field and out-of field components of dose. Each partially in-field organ was divided into two components: (1) in-field component (i.e. the portion of the organ within the 5\% isodose surface) and (2) out-of-field component (i.e. the portion of the organ outside the 5\% isodose surface). These sub-volumes were created using the Boolean operation tools in the TPS. The volume of each organ component was determined using a volume measurement tool in the TPS. The in-field component was taken as the volume-weighted mean dose of the in-field portion of the organ calculated by the TPS, and the out-of-field component was determined using a volume-weighted mean dose from TLD measurements. As previously stated, these calculations were only performed for partially in-field organs where sensitivity analysis revealed that inaccuracies in low-dose regions of the DVH had a substantial effect on the accuracy of the mean organ dose.

Less than one-quarter of the colon, bone marrow and bone surface volumes $(21 \%, 10 \%$ and $11 \%$, respectively) were within the 5\% isodose line. Additionally, significant fractions of these organs were entirely outside of the maximum achievable calculation region of the TPS (i.e. reported as zero dose). Thus, we used the volume-weighted technique to calculate mean doses for the colon, bone marrow and bone surface but did not perform sensitivity analysis because they were obviously partially in-field.

\section{Results}

\subsection{Organ classifications}

For the case described here, in-field organs included the right kidney, liver and stomach.

Out-of-field organs included the brain, eyes, thyroid, heart, breasts, bladder, rectum, prostate and testicles. Partially in-field organs included the left kidney, lungs, spinal cord, esophagus, colon, bone marrow and bone surface.

\subsection{In-field and out-of-field organ doses}

Mean doses for the in-field and out-of-field organs are listed in table 2. Figure 5 shows the TLD-measured doses to the out-of-field organs as a function of the distance from the 5\% isodose line to the center of each organ. The error bars on each data point in figure 5 represent the standard deviation in the measured dose among all TLDs within the organ. Data in figure 5 show that standard deviation depends on the dose gradients, i.e. there is lower deviation in dose for organs that are farther from the treatment field. 


\subsection{Partially in-field organ doses}

Table 3 lists the mean doses to the left kidney, lungs, spinal cord and esophagus calculated using the DVH data from the TPS. In addition, table 3 lists the adjusted mean doses for those organs calculated assuming dosimetric errors of $+60 \%$ and $-60 \%$ for all portions of the DVH with dose less than 5\% of the prescribed dose. Examples of the sensitivity analysis on the accuracy of the low-dose component of the TPS data for partially in-field organs are presented for the lungs and left kidney in figure 4, which shows the original DVH and the DVHs with the low-dose component adjusted assuming dosimetric errors of $+60 \%$ and $-60 \%$. Similar analyses were performed for the spinal cord and esophagus (data not shown). In these figures, there is a discontinuity in the modified DVHs. This exists because we applied the single adjustment of $60 \%$ to all dose values below 5\% of the prescription dose rather than a scaling function which would have resulted in physically valid curves. This single factor was applied to provide the most conservative metric for the sensitivity analysis. These discontinuous DVHs would not be usable for dose assessment, but only to determine whether out-of-field dose measurements are warranted.

For the lungs, spinal cord and esophagus, the adjusted mean doses were within $\pm 5 \%$ of the mean doses reported by the TPS. Therefore, we used DVH data from the TPS alone to assess the mean organ doses to these partially in-field organs. For the left kidney, however, the adjusted mean dose was substantially greater than $5 \%$ of the mean dose reported by the TPS. Therefore, we used the volume-weighted technique to assess the mean dose for the left kidney. The mean TPS-calculated left kidney dose, $51.8 \mathrm{mGy} / \mathrm{Gy}_{\mathrm{Rx}}$ (see table 3), was approximately $13 \%$ lower than the volume-weighted dose, $59.7 \mathrm{mGy} / \mathrm{Gy}_{\mathrm{Rx}}$ (see table 4), calculated from both DVH and measured data. Table 4 also lists the volume-weighted mean doses for the colon, bone marrow and bone surface.

\section{Discussion}

In this study, we established a method to categorize organs as in-field, out-of-field or partially in-field based on isodose inclusion criteria. We used DVHs from the TPS to quantify mean organ doses for in-field organs and measurements in an anthropomorphic phantom to estimate mean doses for out-of-field organs. The study met its objective to evaluate TPS calculations for organs partially within the treatment field and used this evaluation to establish a consistent methodology for determining doses to such organs. We recommend that if inaccuracies in low-dose regions of the DVH will have little effect on the mean organ dose $(< \pm 5 \%)$, TPS data alone may be used to estimate the mean organ dose. But if inaccuracies in low-dose regions of the DVH have a more substantial effect on the mean organ dose ( $>5 \%)$, we suggest using the volume-weighting technique described here to determine the mean dose.

In conventional photon therapy, the out-of-field dose decreases rapidly as the distance from the field edge increases (Stovall et al 1995). For IMRT cases, as in the example presented here, the field edge cannot always be clearly defined. However, the data follow the same trend when the distance from the field edge is replaced by the distance from the $5 \%$ isodose line in analyses. Our data showed that among out-of-field organs those closest to a 5\% isodose line received the highest doses with the greatest variability in dose within the organ. We attribute this pattern to the stray radiation inherent in the treatments. As the distance from the field edge (in conventional therapy) or from the 5\% isodose line (in IMRT) increases, stray radiation contributions change from being composed of both scatter and leakage radiation to leakage radiation only (Fraass and van de Geijn 1983, Stovall et al 1995, Chofor et al 2010). This change is important when designing phantom measurements because there is minimal intra-organ deviation in dose far outside the field $(>20 \mathrm{~cm})$ so fewer TLDs would be required to determine mean organ doses. 
A limitation of the present study was that the criterion for the sensitivity analysis was based on an assumption of a $60 \%$ error in the dose reported by the treatment planning system for doses less than $5 \%$ of the prescription dose. This value was based on data from a previous study for a particular treatment plan calculated using the Eclipse TPS version 8.6 and Varian 2100 Clinac. The exact under- or over-estimation in the out-of-field dose reported for different treatment plans, calculated using different TPSs and linac combinations, is dependent on the particular algorithm used, the commissioning data and the manufacturer. Nonetheless, the $60 \%$ assumption used in this study is a conservative estimate because this represents the maximum deviation that was observed in our previous study. At present there are limited data in the literature regarding out-of-field dose, especially for modern radiation therapy. Recently Xu et al (2008) surveyed the literature for dosimetry studies on external beam radiation treatment and reported 23 studies that considered out-of-field dose from IMRT. The majority of those studies involved measurements and the remainder used Monte Carlo methods. None compared the out-of-field doses to those reported by the treatment planning systems. However, as such data become available, the methodology described in this study can be easily customized with different criterion for the sensitivity analysis for different treatment plan types, planning systems, linacs and commissioning parameters.

This study demonstrated that for some partially in-field organs, the DVH as determined by the TPS underestimated the mean dose by approximately $15 \%$. The focus of this work is on low doses, which may not affect patient care decisions. However, accurate assessment of out-of-field dose is very important in evaluating late effects such as second cancers and for developing dose response models for these low dose effects. For such studies, if part of an organ receives a very large dose and only a small fraction of the volume receives a low dose (such as the lungs in this study), TPS DVHs can be used. However, in scenarios where a majority of the organ volume receives less than $5 \%$ of the prescribed dose and little or none of the volume is in a high-dose gradient (such as the left kidney in this study), it would be prudent to perform a sensitivity analysis, as described here. Results of the sensitivity analysis may then be used to determine whether supplementing TPS calculations with measured data is warranted.

\section{Conclusion}

The method for assessing organ doses throughout the body from photon radiotherapy described here can be used in studies that require accurate knowledge of a wide range of doses from both primary and stray radiation. Such broad information will be of particular use in studies of radiation-induced late effects, which require accurate knowledge of doses to in-field, outof-field and partially in-field organs to predict the risk to organs throughout the body. For example, the methodology established in this study for categorizing organs and determining doses to partially in-field organs may be applied in comparative effectiveness research studies that evaluate both acute and late effects from different radiotherapy treatment techniques.

\section{Acknowledgments}

We would like to thank Kathryn B Carnes and Markeda L Wade for their assistance in preparing and editing this manuscript. This work was supported in part by the National Cancer Institute (awards 5K01CA125204-04 and 1R01CA131463-01A) and by the Fogarty International Center (award K01TW008409). The content in this manuscript is solely the responsibility of the authors and does not necessarily represent the official views of the sponsors. 


\section{References}

Almond PR, Biggs PJ, Coursey BM, Hanson WF, Huq MS, Nath R, Rogers DWO. Aapm's TG-51 protocol for clinical reference dosimetry of high-energy photon and electron beams. Med. Phys 1999;26:1847-70. [PubMed: 10505874]

Aspradakis MM, Morrison RH, Richmond ND, Steele A. Experimental verification of convolution/ superposition photon dose calculations for radiotherapy treatment planning. Phys. Med. Biol 2003;48:2873-93. [PubMed: 14516106]

Bednarz B, Xu XG. Monte Carlo modeling of a 6 and 18 MV Varian clinac medical accelerator for infield and out-of-field dose calculations: development and validation. Phys. Med. Biol 2009;54:N4357. [PubMed: 19141879]

Bhatia S, et al. Late Effects Study Group. High risk of subsequent neoplasms continues with extended follow-up of childhood Hodgkin's disease: report from the late effects study group. J. Clin. Oncol 2003;21:4386-94. [PubMed: 14645429]

Boice JD Jr, Day NE, Andersen A, Brinton LA, Brown R, Choi NW, Clarke EA, Coleman MP, Curtis RE, Flannery JT. Second cancers following radiation treatment for cervical cancer. An international collaboration among cancer registries. J. Natl Cancer Inst 1985;74:955-75. [PubMed: 3858584]

Carr ZA, Kleinerman RA, Stovall M, Weinstock RM, Griem ML, Land CE. Malignant neoplasms after radiation therapy for peptic ulcer. Radiat. Res 2002;157:668-77. [PubMed: 12005546]

Chofor N, Harder D, Ruhmann A, Willborn KC, Wiezorek T, Poppe B. Experimental study on photonbeam peripheral doses, their components and some possibilities for their reduction. Phys. Med. Biol 2010;55:4011-27. [PubMed: 20577041]

Das IJ, Cheng CW, Watts RJ, Ahnesjo A, Gibbons J, Li XA, Lowenstein J, Mitra RK, Simon WE, Zhu TC. Accelerator beam data commissioning equipment and procedures: report of the TG-106 of the therapy physics committee of the AAPM. Med. Phys 2008;35:4186-215. [PubMed: 18841871]

Followill DS, Evans DR, Cherry C, Molineu A, Fisher G, Hanson WF, Lbbott GS. Design, development, and implementation of the radiological physics center's pelvis and thorax anthropomorphic quality assurance phantoms. Med. Phys 2007;34:2070-6. [PubMed: 17654910]

Fraass BA, van de Geijn J. Peripheral dose from megavolt beams. Med. Phys 1983;10:809-18. [PubMed: 6419031]

Goske MJ, et al. The image gently campaign: working together to change practice. Am. J. Roentgenol 2009;190:273-4. [PubMed: 18212208]

Howell RM, Scarboro SB, Kry SF, Yaldo Z. Accuracy of out-of-field dose calculations by a commercial treatment planning system. Phys. Med. Biol 2010;55:6999-7008. [PubMed: 21076191]

Ibbott GS, Molineu A, Followill DS. Independent evaluations of IMRT through the use of an anthropomorphic phantom. Technol. Cancer Res. Treat 2006;5:481-7. [PubMed: 16981790]

ICRU. ICRU Report No 42. International Commission on Radiation Units and Measurements; Bethesda, MD: 1987. Use of computers in external beam radiotherapy procedures with high energy photons and electrons.

Kirby TH, Hanson WF, Gastorf RJ, Chu CH, Shalek RJ. Mailable tld system for photon and electron therapy beams. Int. J. Radiat. Oncol. Biol. Phys 1986;12:261-5. [PubMed: 3949577]

Kirby TH, Hanson WF, Johnston DA. Uncertainty analysis of absorbed dose calculations from thermoluminescence dosimeters. Med. Phys 1992;19:1427-33. [PubMed: 1461205]

Klein EE, Maserang B, Wood R, Mansur D. Peripheral doses from pediatric IMRT. Med. Phys 2006;33:2525-31. [PubMed: 16898456]

Kry SF, Titt U, Pèonisch F, Followill D, Vassiliev ON, White RA, Mohan R, Salehpour M. A Monte Carlo model for calculating out-of-field dose from a Varian $6 \mathrm{MV}$ beam. Med. Phys 2006;33:4405-13. [PubMed: 17153419]

Lin HM, Teitell MA. Second malignancy after treatment of pediatric Hodgkin disease. J. Pediatr. Hematol. Oncol 2005;27:28-36. [PubMed: 15654275]

Meeks SL, Paulino AC, Pennington EC, Simon JH, Skwarchuk MW, Buatti JM. In vivo determination of extra-target doses received from serial tomotherapy. Radiother. Oncol 2002;63:217-22. [PubMed: 12063012] 
Newhauser WD, et al. The risk of developing a second cancer after receiving craniospinal proton irradiation. Phys. Med. Biol 2009;54:2277-91. [PubMed: 19305036]

Reft CS, Runkel-Muller R, Myrianthopoulos L. In vivo and phantom measurements of the secondary photon and neutron doses for prostate patients undergoing 18 MV IMRT. Med. Phys 2006;33:3734-42. [PubMed: 17089839]

Ron E. Cancer risks from medical radiation. Health Phys 2003;85:47-59. [PubMed: 12852471]

Rubino C, Adjadj E, Guerin S, Guibout C, Shamsaldin A, Dondon MG, Valteau-Couanet D, Hartmann O, Hawkins M, de Vathaire F. Long-term risk of second malignant neoplasms after neuroblastoma in childhood: role of treatment. Int. J. Cancer 2003a;107:791-6. [PubMed: 14566829]

Rubino C, de Vathaire F, Dottorini ME, Hall P, Schvartz C, Couette JE, Dondon MG, Abbas MT, Langlois C, Schlumberger M. Second primary malignancies in thyroid cancer patients. Br. J. Cancer 2003b;89:1638-44. [PubMed: 14583762]

Sievinen, J.; Ulmer, W.; Kaissl, W. Varian Medical Systems white paper. Varian Medical Systems; Palo Alto, CA: 2005. AAA photon dose calculation model in eclipse.

Sigurdson AJ, et al. Primary thyroid cancer after a first tumour in childhood (the childhood cancer survivor study): a nested case-control study. Lancet 2005;365:2014-23. [PubMed: 15950715]

Stovall M, Blackwell CR, Cundiff J, Novack DH, Palta JR, Wagner LK, Webster EW, Shalek RJ. Fetal dose from radiotherapy with photon beams: report of AAPM Radiation Therapy Committee Task Group no 36. Med. Phys 1995;22:63-82. [PubMed: 7715571]

Stovall M, Smith SA, Rosenstein M. Tissue doses from radiotherapy of cancer of the uterine cervix. Med. Phys 1989;16:726-33. [PubMed: 2509867]

Stovall M, Weathers R, Kasper C, Smith SA, Travis L, Ron E, Kleinerman R. Dose reconstruction for therapeutic and diagnostic radiation exposures: use in epidemiological studies. Radiat. Res 2006;166:141-57. [PubMed: 16808603]

Taddei PT, Howell RM, Scarboro SB, Krishnan S, Mirkovic D, Newhauser WD. Risk of second malignant neoplasm following proton versus intensity modulated photon radiotherapy for hepatocellular carcinoma. Phys. Med. Biol 2010;55:7055-65. [PubMed: 21076199]

Van Esch A, Tillikainen L, Pyykkonen J, Tenhunen M, Helminen H, Siljamaki S, Alakuijala J, Paiusco M, Lori M, Huyskens DP. Testing of the analytical anisotropic algorithm for photon dose calculation. Med. Phys 2006;33:4130-48. [PubMed: 17153392]

Xu XG, Bednarz B, Paganetti H. A review of dosimetry studies on external-beam radiation treatment with respect to second cancer induction. Phys. Med. Biol 2008;53:R193-241. [PubMed: 18540047] 


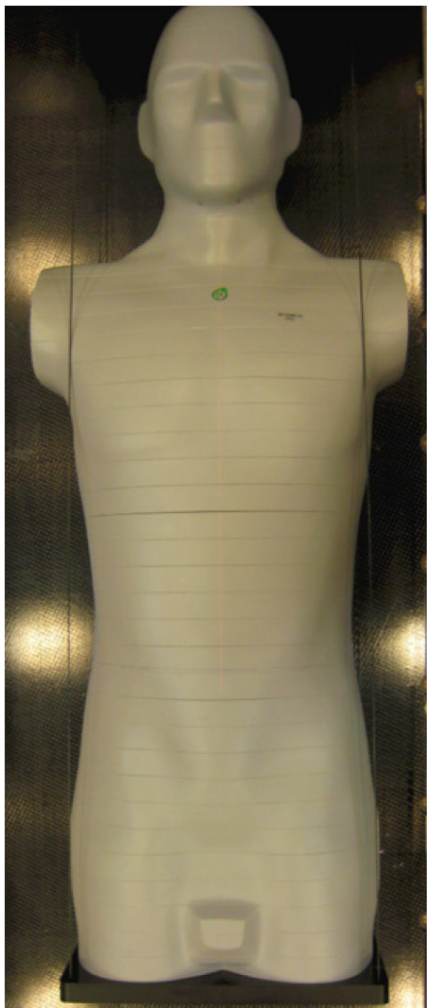

Figure 1.

Photograph of ATOM male dosimetry phantom (CIRS, Inc., Norfolk, VA) used in this study. 


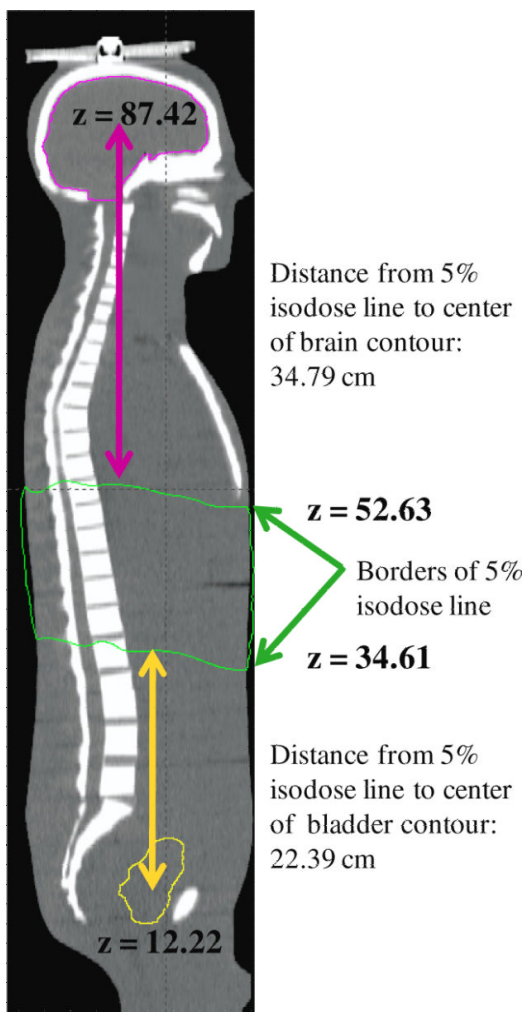

Figure 2.

Illustration of how the distance between the center of each out-of-field organ and the border of the nearest $5 \%$ isodose line was determined, showing the superior and inferior 5\% isodose lines (green) and the distances from these lines for organs above (brain, pink line) and below (bladder, yellow line) the lines. 


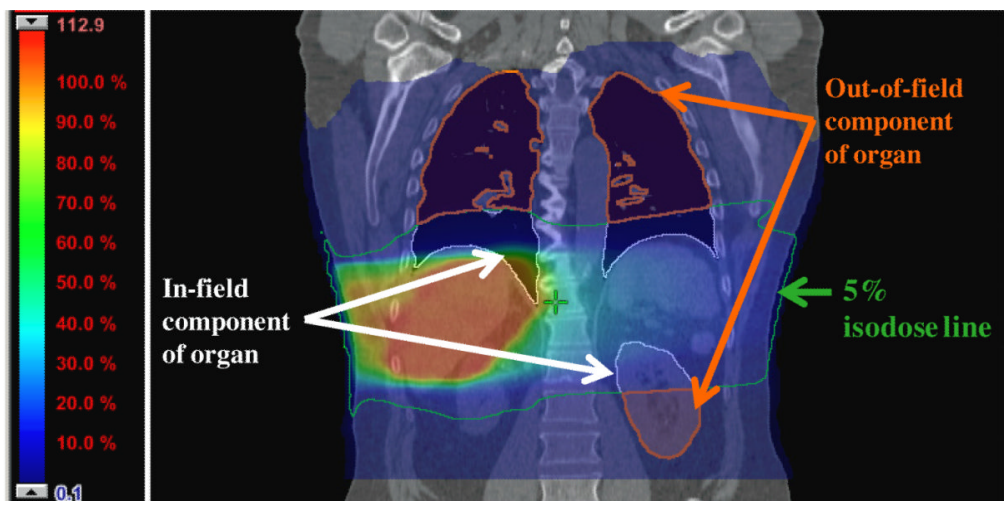

Figure 3.

Frontal view of the patient CT dataset at the isocenter plane with the dose displayed as a color wash from the maximum dose of $112.9 \%$ to $0.1 \%$ of the prescribed dose and showing two partially in-filed organs, the lungs and the left kidney. The 5\% isodose line is outlined using a green contour and out-of-filed and in-field portions of the lungs and left kidney shown in orange and white contours, respectively. 

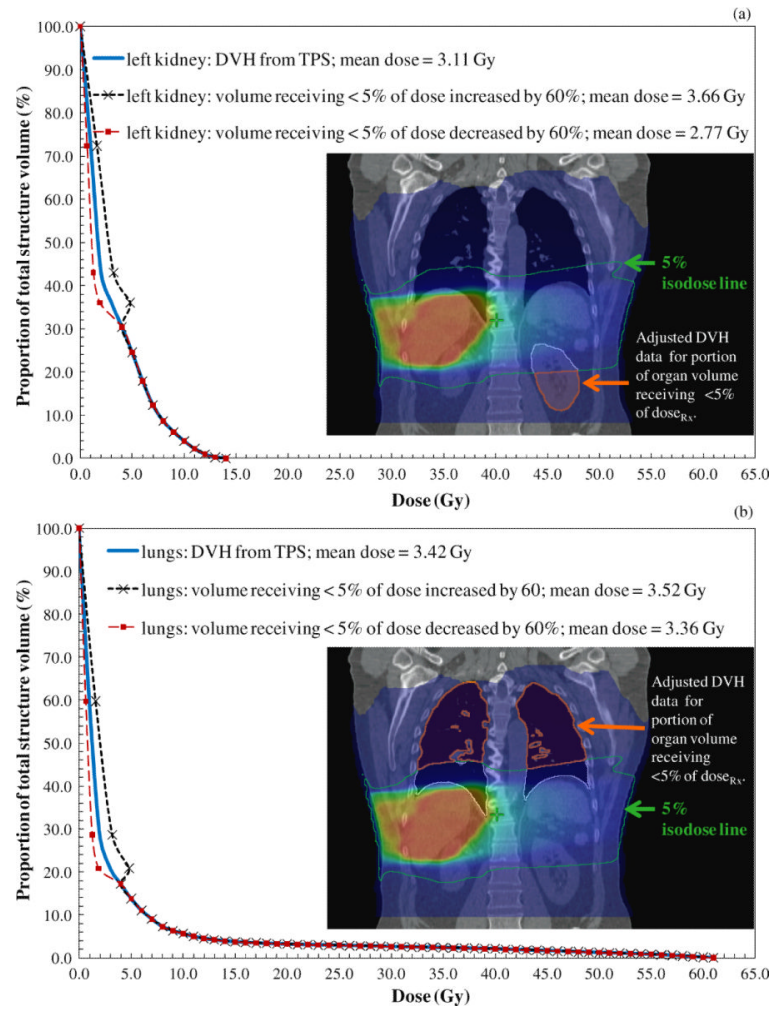

Figure 4.

TPS data for the left kidney (a) lungs (b) and esophagus (c) showing the original DVH calculated by the TPS and the adjusted DVHs for regions receiving less than $5 \%$ of the prescribed dose (i.e. low-dose regions) adjusted assuming dosimetric errors of $+60 \%$ and $-60 \%$. 


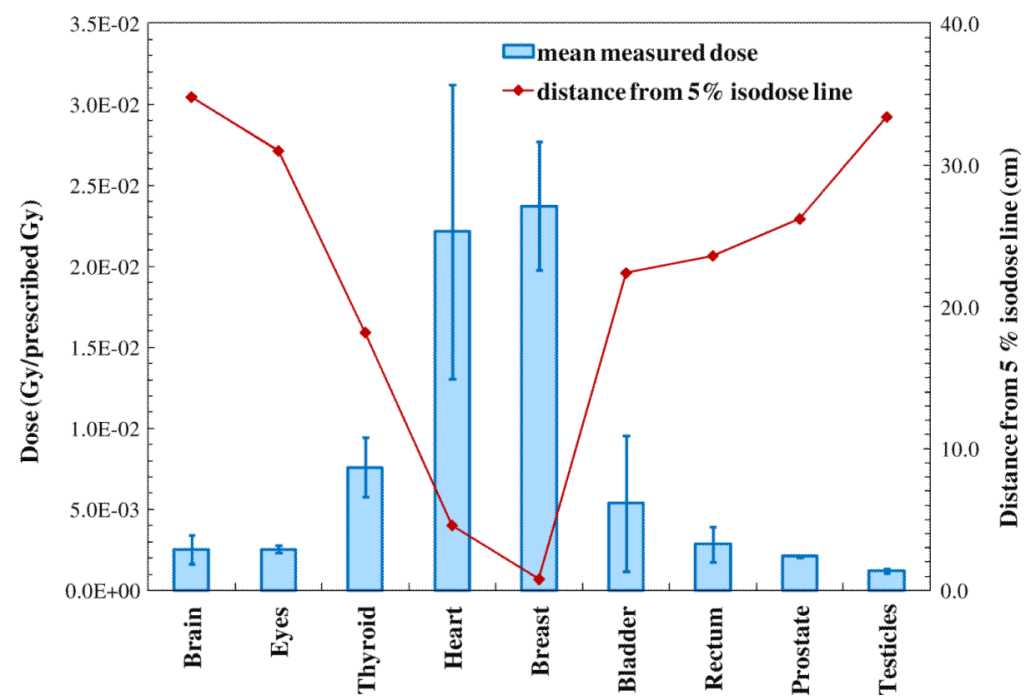

Figure 5.

TLD-measured mean organ dose for the out-of-field organs (primary $y$-axis) and the relationship between the mean dose of the distance from the center of each organ to the nearest 5\% isodose line (secondary $y$-axis). The error bars on each mean dose data point represent standard deviations in measured doses among all TLDs within the organs. 


\section{Table 1}

Normal-tissue objectives used for treatment planning and corresponding values from the final actual treatment plan. $V_{x}$ represents the percentage of volume receiving $x$ Gy of dose, and D95 represents the dose received by $95 \%$ of the volume.

\begin{tabular}{llll}
\hline Structure & Dosimetric parameter & Prescribed & Actual \\
\hline Planning target volume & $D_{95}$ & $60 \mathrm{~Gy}$ & $60.0 \mathrm{~Gy}$ \\
Liver-clinical target volume & $V_{30}$ & $<40 \%$ & $21.2 \%$ \\
Right (ipsilateral) kidney & $V_{20}$ & $<50 \%$ & $50.9 \%$ \\
Left (contralateral) kidney & $V_{20}$ & $<30 \%$ & $0.0 \%$ \\
\hline
\end{tabular}


Table 2

Mean organ absorbed dose values and standard deviations (variation about the mean) for out-of-field organs from TLD measurements and for in-field organs from TPS calculations.

\begin{tabular}{|c|c|c|}
\hline Organ & Mean dose $\left(\mathbf{G y} / \mathbf{G y}_{\mathbf{R x}}\right)$ & Standard deviation \\
\hline \multicolumn{3}{|l|}{ Out-of-field } \\
\hline Brain & $2.5 \times 10^{-3}$ & $0.9 \times 10^{-3}$ \\
\hline Eyes & $2.5 \times 10^{-3}$ & $0.2 \times 10^{-3}$ \\
\hline Thyroid & $7.6 \times 10^{-3}$ & $1.8 \times 10^{-3}$ \\
\hline Heart & $22.1 \times 10^{-3}$ & $9.0 \times 10^{-3}$ \\
\hline Breast & $23.7 \times 10^{-3}$ & $3.9 \times 10^{-3}$ \\
\hline Bladder & $5.3 \times 10^{-3}$ & $4.2 \times 10^{-3}$ \\
\hline Rectum & $2.8 \times 10^{-3}$ & $1.1 \times 10^{-3}$ \\
\hline Prostate & $2.1 \times 10^{-3}$ & $0.7 \times 10^{-3}$ \\
\hline Testicles & $1.2 \times 10^{-3}$ & $0.2 \times 10^{-3}$ \\
\hline \multicolumn{3}{|l|}{ In-field } \\
\hline Right kidney & $4.54 \times 10^{-1}$ & $3.73 \times 10^{-1}$ \\
\hline Spinal cord & $2.35 \times 10^{-1}$ & $1.93 \times 10^{-1}$ \\
\hline Esophagus & $1.87 \times 10^{-1}$ & $2.62 \times 10^{-1}$ \\
\hline Liver-CTV & $3.38 \times 10^{-1}$ & $2.91 \times 10^{-1}$ \\
\hline Stomach & $1.75 \times 10^{-1}$ & $0.90 \times 10^{-1}$ \\
\hline
\end{tabular}




\section{Table 3}

Mean doses for partially in-field organs from DVHs calculated by the TPS and the retrospectively adjusted mean doses computed assuming dosimetric errors of $+60 \%$ and $-60 \%$ for all portions of the DVH with dose less than $5 \%$ of the prescribed dose.

\begin{tabular}{llc}
\hline Organ: calculation method & Mean dose $\left(\mathbf{G y} / \mathbf{G y}_{\mathbf{R x}}\right)$ & Difference from DVH TPS-calculated mean \\
\hline Spinal cord: TPS & $2.35 \times 10^{-1}$ & - \\
Spinal cord: adjusted +60\% dose error & $2.40 \times 10^{-1}$ & $1.8 \%$ \\
Au: Spinal cord: adjusted -60\% dose error & $2.33 \times 10^{-1}$ & $1.1 \%$ \\
Esophagus: TPS & $1.87 \times 10^{-1}$ & - \\
Esophagus: adjusted +60\% dose error & $1.88 \times 10^{-1}$ & $0.8 \%$ \\
Esophagus: adjusted -60\% dose error & $1.86 \times 10^{-1}$ & $0.5 \%$ \\
Left kidney: TPS & $0.52 \times 10^{-1}$ & - \\
Left kidney: adjusted +60\% dose error & $0.61 \times 10^{-1}$ & $17.3 \%$ \\
Left kidney: adjusted -60\% dose error & $0.46 \times 10^{-1}$ & $11.5 \%$ \\
Lungs: TPS & $0.57 \times 10^{-1}$ & - \\
Lungs: adjusted $+60 \%$ dose error & $0.59 \times 10^{-1}$ & $3.5 \%$ \\
Lungs: adjusted $-60 \%$ dose error & $0.56 \times 10^{-1}$ & $1.8 \%$ \\
\hline
\end{tabular}




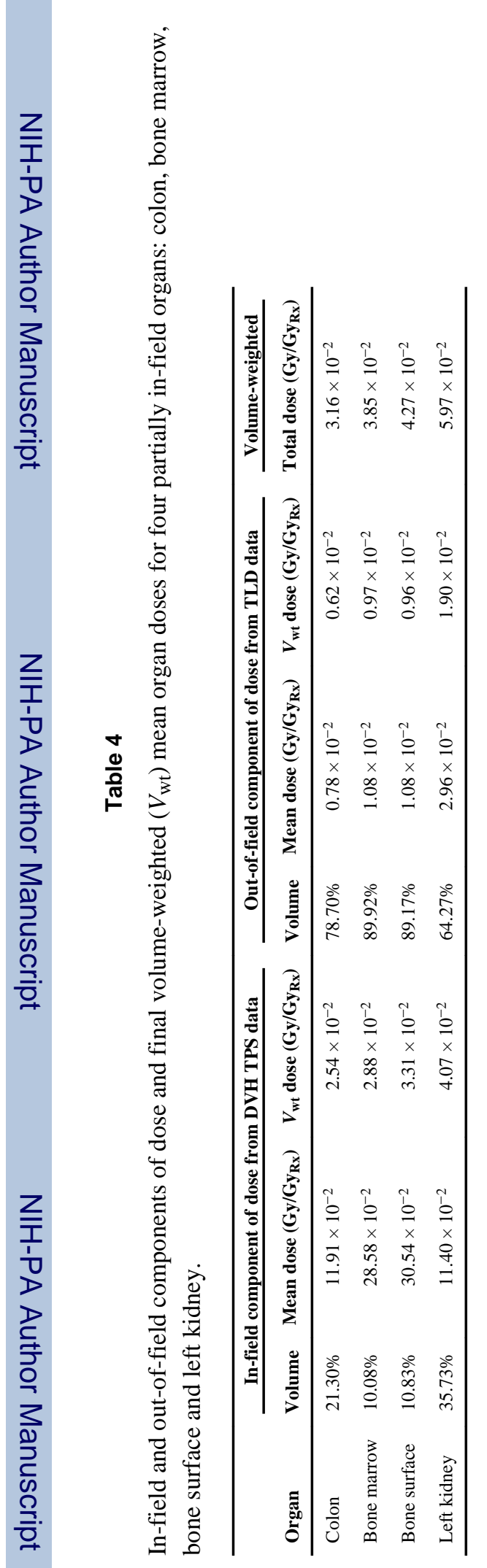

